\title{
Horseshoes for autonomous Hamiltonian systems using the Melnikov integral
}

\author{
CLARK ROBINSON \\ Northwestern University, Evanston, IL 60208, USA
}

\begin{abstract}
This paper applies the Melnikov method to autonomous perturbations of completely integrable Hamiltonian systems. The forcing of the perturbed system is caused by internal oscillations which are not necessarily decoupled. A unified treatment is presented which relates some results of Holmes and Marsden with a result of Lerman and Umanskii. It is also shown that two forms of the Melnikov function by integrals are in fact equal.
\end{abstract}

\section{Statement of results}

During the last 20 years, there has been much interest and progress in determining hyperbolic horseshoes for perturbations of Hamiltonian systems. In particular, the existence of such horseshoes implies that the system is not integrable and has chaotic motion. One basic method was introduced by Melnikov [13] using ideas which go back to Poincaré and is often called the Melnikov method or Poincaré-MelnikovArnold method. See [12] for a discussion of the various contributions of Arnold and others. Often in these references, the perturbation is an external time periodic perturbation or at least is the effect of an oscillation which decouples (as in the restricted three-body problem considered by Silnikov.) Recently, there have been several papers applying Melnikov's method to autonomous Hamiltonian systems where the perturbation forcing is internal (rather than an external time-dependent forcing) and the oscillation does not decouple. In particular, there are the three papers by Holmes and Marsden (see [8], [9] and [10]) which study orbits homoclinic to hyperbolic closed orbits and quasi-periodic motion on tori, and the paper by Lerman and Umanskii [11] which studies orbits homoclinic to a hyperbolic fixed point. The paper by Gruendler [6] has some related results on higher-dimensional systems but with external time periodic forcing.

This paper presents a unified treatment of these results and gives an alternative formula to that given by Holmes and Marsden for the the general case for systems with an $S^{1}$ symmetry. In some of these cases the integrals are only conditionally convergent. We indicate carefully how the sequence of limits of integration must be chosen so that the result has the correct dynamic meaning. Salem et al. also noticed that the limits must be carefully chosen (see [19] or [12]).

In either case we consider a Hamiltonian with two degrees of freedom, four total dimensions, with Hamiltonian function

$$
H^{\varepsilon}(z)=H_{0}(z)+\varepsilon H_{1}(z)+\varepsilon^{2} H_{2}(z)+\cdots \text {. }
$$


To form the associated vector field or differential equation let

$$
J_{2}=\left(\begin{array}{rr}
0 & 1 \\
-1 & 0
\end{array}\right)
$$

be the two by two matrix and

$$
J=\left(\begin{array}{cc}
J_{2} & 0 \\
0 & J_{2}
\end{array}\right)
$$

be the standard four by four skew symmetric non-degenerate matrix. For any real-valued function $G(z)$ let $\dot{z}=J \nabla G=X_{G}$ be the associated Hamiltonian vector field. In particular, for $H^{\varepsilon}$, let

$$
\begin{aligned}
\dot{z} & =J \nabla H^{\varepsilon}=X_{H^{\varepsilon}}(z)=X^{\varepsilon}(z) \\
& =J \nabla H_{0}+\varepsilon J \nabla H_{1}+\cdots=X_{0}(z)+\varepsilon X_{1}(z)+\cdots .
\end{aligned}
$$

We let $z=\phi\left(t, z_{0}, \varepsilon\right)$ be the solutions of $\dot{z}=X^{\varepsilon}(z)$ with $\phi\left(0, z_{0}, \varepsilon\right)=z_{0}$. Also, $\phi_{0}\left(t, z_{0}\right)=\phi\left(t, z_{0}, 0\right)$. The basic assumption for this paper is that $X_{0}$ has a second real-valued independent integral $F$ :

$D F \cdot X_{0} \equiv 0$ so $F$ is constant on solution of $X_{0}$;

$\nabla F$ and $\nabla H_{0}$ are independent.

(In particular, they are independent on the homoclinic connection $\Gamma$ discussed below.) With this assumption, $F$ is a valid measure of one of the directions in the energy surface $H_{0}^{-1}(h)$ which is transverse to the solutions of $X_{0}$. A Hamiltonian system in dimension four with such a second integral is called completely integrable.

1A. In the first case, we assume $H_{0}$ has a hyperbolic closed orbit $\gamma$ with a homoclinic connection. In other words, at least one branch of the stable manifold $W^{\mathrm{s}}\left(\gamma, H_{0}\right)$ coincides with a branch of the unstable manifold $W^{\mathrm{u}}\left(\gamma, H_{0}\right)$. Let $\Sigma$ be a transversal to the flow and so it is three-dimensional. Assume $H_{0}(\gamma)=0$. The energy surface $H_{0}^{-1}(0)$ is also three-dimensional, and their intersection $\Sigma \cap H_{0}^{-1}(0)$ is twodimensional. Since $X_{0}$ has a second integral, if $W^{\mathrm{u}}\left(\gamma, H_{0}\right)$ and $W^{\mathrm{s}}\left(\gamma, H_{0}\right)$ intersect, they coincide on components of $W^{\mathrm{s}}\left(\gamma, H_{0}\right)-\gamma$ and $W^{\mathrm{u}}\left(\gamma, H_{0}\right)-\gamma$. Let $\Gamma$ be one such component of $\left[W^{\mathrm{s}}\left(\gamma, H_{0}\right)-\gamma\right] \cap W^{\mathrm{u}}\left(\gamma, H_{0}\right)$. (Note that it can be shown that $F$ and $H_{0}$ are necessarily dependent on $\gamma$ since $F$ is constant on $W^{s}\left(\gamma, H_{0}\right)$, $W^{\mathrm{u}}\left(\gamma, H_{0}\right)$ and $\left.\gamma.\right)$

Now, for $z$ in $\Gamma$, let $N_{z}$ be a plane transverse to $\Gamma$ at $z$ (or even normal). Note that $\Gamma$ is two-dimensional so a plane is transverse in the total space. For $\varepsilon>0$, let $z^{\mathrm{u}}(\varepsilon)$ be a point in $W^{\mathrm{u}}\left(\gamma_{\varepsilon}, H_{\varepsilon}\right) \cap N_{z}$ where the perturbed unstable manifold intersects $N_{z}$. Such a choice of points can be made smoothly in $\varepsilon$, because $\gamma_{\varepsilon}$ varies smoothly as a function of $\varepsilon$ since $\gamma$ is hyperbolic and $W^{\mathrm{u}}\left(\gamma_{\varepsilon}, H^{\varepsilon}\right)$ varies smoothly on compact subsets. Similarly define $z^{\mathrm{s}}(\varepsilon)=W^{\mathrm{s}}\left(\gamma_{\varepsilon}, H^{\varepsilon}\right) \cap N_{z}$. Now $F$ can be used to measure the relative displacement of $W^{\mathrm{s}}\left(\gamma_{\varepsilon}, H^{\varepsilon}\right)$ and $W^{\mathrm{u}}\left(\gamma_{\varepsilon}, H^{\varepsilon}\right)$; define

$$
M(z)=\left.\frac{\partial}{\partial \varepsilon}\left\{F\left(z^{\mathrm{u}}(\varepsilon)\right)-F\left(z^{\mathrm{s}}(\varepsilon)\right)\right\}\right|_{\varepsilon=0} .
$$

If $M(z)$ has a non-degenerate zero as $z$ varies on $\Gamma$, then $W^{\mathrm{s}}\left(\gamma_{\varepsilon}, H_{\varepsilon}\right)$ and $W^{\mathrm{u}}\left(\gamma_{\varepsilon}, H_{\varepsilon}\right)$ have a transverse intersection nearby because $M$ measures the lowest-order separ- 
ation of these manifolds. Theorem 6 discusses the case when $M$ merely changes sign at a point $z_{0}$.

The following theorem gives a formula to calculate $M$ in terms of an integral along the orbits of the unperturbed system. It is the basic fact which makes the integral applicable.

THEOREM 1. Assume the Hamiltonian vector field for $H_{0}$ has a closed orbit $\gamma$ with a homoclinic connection $\Gamma$. Further, assume that $H_{0}$ is completely integrable with a second integral $F$ which is independent from $H_{0}$ on $\Gamma$. Then

$$
M(z)=\lim _{j \rightarrow \infty} \int_{-T_{j}^{*}}^{T_{j}}\left(D F \cdot X_{1}\right)_{\phi_{0}(t, z)} d t
$$

if $T_{j}$ and $T_{j}^{*}$ are chosen such that $\lim _{j \rightarrow 0}\left|\phi_{0}\left(T_{j}, z\right)-\phi_{0}\left(-T_{j}^{*}, z\right)\right|=0$. Thus $\phi_{0}\left(T_{j}, z\right)$ and $\phi_{0}\left(-T_{j}^{*}, z\right)$ converge to the same point on the closed orbit $\gamma$. If DF $\cdot X_{1}=0$ for points on $\gamma$, then the improper integral converges absolutely. In particular, if $\gamma$ remains a closed orbit when $\varepsilon>0$ (and so $X_{1}$ is parallel to $X_{0}$ on $\gamma$ ), then the integral converges absolutely.

The proof of this theorem is similar to those in the literature, but is given in $\S 2$ for completeness and because it is not very long. The limits of integration need to be chosen as specified in the theorem because the closed orbit can move as $\varepsilon$ becomes positive. The proof shows that

$$
M(z)=\int_{-T_{j}^{*}}^{T_{j}}\left(D F \cdot X_{1}\right)_{\phi_{0}(t, z)} d t+R\left(-T_{j}^{*}, T_{j}\right),
$$

where

$$
R\left(-T_{j}^{*}, T_{j}\right)=\frac{\partial}{\partial \varepsilon} F \circ \phi\left(-T_{j}^{*}, z^{\mathrm{u}}(\varepsilon), \varepsilon\right)-\frac{\partial}{\partial \varepsilon} F \circ \phi\left(T_{j}, z^{\mathrm{s}}(\varepsilon), \varepsilon\right) .
$$

Lemma 2.1 proves that as $T_{j}$ and $T_{j}^{*} \rightarrow \infty, R\left(-T_{j}^{*}, T_{j}\right)$ converges to the difference of the infinitesimal displacement of $\gamma_{\varepsilon}$ near $\phi_{0}\left(-T_{j}^{*}, z\right)$ and $\phi_{0}\left(T_{j}, z\right)$ as measured by $F$. If these converge to the same point, then the remainder goes to zero. The following example taken from [10, example 6.5] shows that this is indeed the case for a particular Hamiltonian.

Example 1.5. Let

$$
H^{\varepsilon}(r, p, \theta, I)=p^{2} / 2+U(r)+I^{2} /\left(2 r^{2}\right)+\varepsilon \sin \theta .
$$

This rises from a slightly asymmetric central force problem. Since $\dot{I}=-\varepsilon \cos \theta$, $F=I$ can be taken as the second integral of $H_{0}$. Choose $U(r)$ with a simple maximum and $I_{0}$ such that the effective potential $U(r)+I_{0}^{2} /\left(2 r^{2}\right)$ has a minimum at $r_{-}$and a maximum at $r_{+}$. Then $H_{0}$ has a saddle closed orbit with $r=r_{+}, v=0$, $I=I_{0}$ and $\theta$ varying. It also has a homoclinic orbit $\left(r_{0}(t), p_{0}(t), \theta_{0}(t)+\theta^{0}, I_{0}\right)$ with $p_{0}(0)=0, \theta_{0}(0)=0, r_{0}(-t)=r_{0}(t)$ and $\theta_{0}(-t)=-\theta(t)$ for any choice of the initial condition $\theta^{0}$. Then $D F \cdot X_{H_{1}}=-\cos \left(\theta_{0}(t)+\theta^{0}\right)$ and

$$
M\left(\theta^{0}\right)=-\lim \int_{-T_{i}^{*}}^{T_{i}} \cos \left(\theta_{0}(t)+\theta^{0}\right) d t .
$$


Note that any orbit on $\Gamma$ can be realized by a suitable choice of $\theta^{0}$, so it is the only variable used for the function $M$. Let $\theta_{j}=\theta_{0}\left(T_{j}\right)$ and $-\theta_{j}^{*}=\theta_{0}\left(-T_{j}^{*}\right)$. Since $\dot{\theta}=I_{0} / r^{2}$,

$$
\begin{aligned}
M\left(\theta^{\circ}\right)= & -\cos \theta^{0} \lim _{j} \frac{1}{I_{0}} \int_{-\theta_{j}^{*}}^{\theta_{j}} r_{0}(t)^{2} \cos \theta d \theta \\
& +\sin \theta^{0} \lim _{j} \frac{1}{I_{0}} \int_{-\theta_{j}^{*}}^{\theta_{j}} r_{0}(t)^{2} \sin \theta d \theta,
\end{aligned}
$$

where $r_{0}(t)$ is evaluated at the $t$ corresponding to $\theta$. The limits need to be chosen so that $\left|\theta_{j}+\theta^{0}-\left(-\theta_{j}^{*}+\theta^{0}\right)\right|=\left|\theta_{j}+\theta_{j}^{*}\right|$ goes to zero modulo $2 \pi$. Here, this is most easily done by taking $\theta_{j}=2 \pi j$ and $-\theta_{j}^{*}=-2 \pi j$. Since $r_{0}(t)^{2} \sin \theta$ is an odd function, the limit of the second integral is zero. (This is easier to see if the limits are $\pm 2 \pi j$, but is true in any case using the fact that $r_{0}(t) \rightarrow r_{+}$as $t \rightarrow \pm \infty$.) The limit of the first integral is non-zero for generic choices of $U(r)$. Note that the limit of each integral can vary by $\pm r_{+}^{2}$ by choosing the limits $\theta_{j}$ and $-\theta_{j}^{*}$ incorrectly. Finally, integration by parts on the first integral yields

$$
M\left(\theta^{0}\right)=\cos \theta^{0}\left(\frac{2}{I_{0}}\right) \int_{-\infty}^{\infty} r_{0}(t) p_{0}(t) \sin \theta_{0}(t) d t,
$$

since $r_{0}\left(T_{j}\right)^{2} \sin \theta_{j}-r_{0}\left(-T_{j}^{*}\right)^{2} \sin \left(-\theta_{j}^{*}\right) \rightarrow 0$. This integral converges absolutely because $p_{0}(t)$ goes to zero exponentially. This answer is just the negative of the answer in [10] as theorem 3 below shows it must be.

1B. The second case we discuss was considered by Lerman and Umanskii [11]. It assumes the system has hyperbolic fixed point $P$. They prove the comparable result which we state as theorem 2 . We wish to thank P. Holmes for pointing out this result to us after he had seen a version of theorem 1 used in some joint work with J. Koiller.

TheOREM 2. Assume $H_{0}$ is completely integrable with second integral $F$ and has a hyperbolic fixed point $P$ with non-empty homoclinic connection $\Gamma=\left[W^{s}\left(P, H_{0}\right)-\{P\}\right] \cap$ $W^{\mathrm{u}}\left(P, H_{0}\right)$. Assume the integral $F$ is independent of $H_{0}$ along $\Gamma$. Then, for $z \in \Gamma$, the formula for $M(z)$ is as given in theorem 1 and the integral always converges absolutely.

The proof of this theorem is exactly the same as theorem 1, so it follows from $\S 2$. The reason that the integral converges absolutely is that there is only one point on $P$, so the limit

$$
\lim _{t \rightarrow 0}\left|\phi_{0}(t, z)-\phi_{0}(-t, z)\right|=0
$$

without taking sequences of times.

1C. Next we want to relate this formula to the one derived by Holmes and Marsden in the most general case with $S^{1}$ symmetry [10, p. 299ff]. Such a symmetry naturally gives a second integral $I$. In fact, by using the related action angle coordinates, they consider $z=(y, \theta, I)$ and Hamiltonians

$$
H^{\varepsilon}(z)=H_{0}(y, I)+\varepsilon H_{1}(z)+\cdots,
$$

where $H_{0}$ is independent of $\theta$. If the motion is restricted to a single energy surface, then $\theta$ can be considered a new time and the system as having a time-dependent 
perturbation by $X_{1}$, i.e. a $\theta$-dependent perturbation. Then the usual Melnikov integral applies. They show the resulting formulae are as follows. Let $\Omega=\partial H_{0} / \partial I$ and

$$
Y_{1}=\left(\begin{array}{l}
\frac{\partial}{\partial y_{2}}\left(H_{1} / \Omega\right) \\
-\frac{\partial}{\partial y_{1}}\left(H_{1} / \Omega\right)
\end{array}\right)
$$

If $X_{0}$ has a closed orbit with homoclinic connection, and if $z_{0}=\left(y_{0}, \theta_{0}, I_{0}\right)$ is the initial condition, then they get a Melnikov integral

$$
M^{*}\left(z_{0}\right)=\int_{-\infty}^{\infty}\left(D H_{0} Y_{1}\right)_{\phi_{0}\left(t, z_{0}\right)} d t .
$$

This integral is always absolutely convergent because $D H_{0}$ vanishes at points on the closed orbit $\gamma$ so $D H_{0} \cdot Y_{1}=0$ at such points. Our next theorem relates their function $M^{*}(z)$, which can be calculated with formula (1.6), with our function $M(z)$, which can be calculated by formula (1.4).

THEOREM 3. Under the conditions above, $M(z)=-M^{*}(z)$.

The proof of this theorem is given in $\$ 3$. The principal step in the proof is integration by parts. Note that because integration by parts involves evaluating a product at the limits of integration in addition to the two integrals, there is no contradiction to the fact that $M^{*}$ converges absolutely and $M$ only conditionally. See example 1.5 above.

Since these two integrals differ by only a sign and both measure the separation of stable and unstable manifolds, either formula can be applied to a given problem. Their integral has the advantage that it is absolutely convergent and may be simpler in some problems. The difficulty is that, to calculate their integral, the system must be converted explicitly into action angle coordinates, the $\theta$ and $I$, in order to calculate $\Omega=\partial H_{0} / \partial I$. In some problems this difficult to attain. In these cases $M(p)$ may be the better choice. Correspondence with J. Koiller about such problems is what motivated much of this work.

1D. Next, Holmes and Marsden considered higher degrees of freedom in the case with a $\left(S^{1}\right)^{n}$ symmetry [9]. In particular, they assumed $\theta=\left(\theta_{1}, \ldots, \theta_{n}\right)$ and $I=\left(I_{1}, \ldots, I_{n}\right)$, and that not only is $H_{0}$ independent of $\theta$, but also that

$$
H^{\varepsilon}(q, p, \theta, I)=F(q, p)+\sum_{j=1}^{n} G_{j}\left(I_{j}\right)+\varepsilon H_{1}(q, p, \theta, I) .
$$

They assume $F$ has a homoclinic orbit in $(q, p)$-space to a hyperbolic fixed point $y^{*}=\left(q^{*}, p^{*}\right)$. As a result, $H_{0}=F+\sum G_{j}$ has an invariant $n$-torus $\left\{\left(q^{*}, p^{*}, \theta, I_{0}\right)\right\}=$ $T_{0}^{n}$, where $I_{0}=\left(I_{10}, \ldots, I_{n 0}\right)$ is chosen so the motion is quasi-periodic. This torus has two normal directions in which it is hyperbolic and one component of the stable and unstable manifolds coincides,

$$
\left[W^{\mathrm{s}}\left(T_{0}^{n}, H_{0}\right)-T_{0}^{n}\right] \cap W^{\mathrm{u}}\left(T_{0}^{n}, H_{0}\right)=\Gamma \text {. }
$$

This system is completely integrable for $\varepsilon=0$ with integrals $\left(F, I_{1}, \ldots, I_{n-1}\right)$ or $\left(I_{1}, \ldots, I_{n}\right)$ or $\left(F, G_{1}, \ldots, G_{n-1}\right)$ or some variation on these. Together one of these 
sets of integrals measures the distances transverse to

$$
\Gamma=W^{\mathrm{s}}\left(T_{0}^{n}, H_{0}\right) \cap\left[W^{\mathrm{u}}\left(T_{0}^{n}, H_{0}\right)-T_{0}^{n}\right] \quad \text { in } H_{0}^{-1}(h) .
$$

They then form a Melnikov vector using the integrals $\left(I_{1}, \ldots, I_{n-1}, F\right)$. We proceed slightly differently than they did and define this vector-valued function in a manner consistent with the other cases considered above.

Consider

$$
H^{\varepsilon}(z)=H_{0}(z)+\varepsilon H_{1}(z)+\varepsilon^{2} H_{2}(z, \varepsilon),
$$

where $z$ has $2 n+2$ dimensions. We need the following assumptions:

(H1) $H_{0}$ is completely integrable, with integrals $\left\{F_{1}, \ldots, F_{n}\right\}$ in addition to $H_{0}$.

(H2) The Hamiltonian vector field $X_{H_{0}}$ for $H_{0}$ has an invariant $n$-torus $T_{0}^{n}$ on which the motion is quasi-periodic, given by a frequency vector $\omega=\left(\omega_{1}, \ldots, \omega_{n}\right) \in \mathbb{R}^{n}$ satisfying the Diophantine conditions $|(\omega, j)| \geq c \mid j^{-\tau}$, with two positive constants $c>0$ and $\tau>n-1$, for all integer vectors $j \in Z^{n}$ with $|j|=\sum_{\nu=1}^{n}\left|j_{\nu}\right|>0$.

(H3) There are two dimensions normal to $T_{0}^{n}$ in which the motion is hyperbolic (with one contracting direction and one expanding direction). Let $W^{\mathrm{s}}\left(T_{0}^{n}, H_{0}\right)$ and $W^{\mathrm{u}}\left(T_{0}^{n}, H_{0}\right)$ be the $(n+1)$-dimensional exponentially attracting and repelling manifolds for $T_{0}^{n}$. Assume $T_{0}^{n}$ has a homoclinic orbit and let $\Gamma=W^{\mathrm{s}}\left(T_{0}^{n}, H_{0}\right) \cap$ [ $W^{\mathrm{u}}\left(T_{0}^{n}, H_{0}\right)-T_{0}^{n}$ ]. Assume the integrals $F_{j}$ are independent of $H_{0}$ on $\Gamma$. Thus the flow of the vector fields $X_{F_{j}}$ must leave $\Gamma$ invariant, so $\Gamma$ is $(n+1)$-dimensional, i.e. the manifolds intersect on components of $W^{\mathrm{s}}\left(T_{0}^{n}, H_{0}\right)-T_{0}^{n}$ and $W^{\mathrm{u}}\left(T_{0}^{n}, H_{0}\right)-T_{0}^{n}$.

(H4) Let $W^{c}\left(T_{0}^{n}, H_{0}\right)$ be the (local) centre manifold of dimension $2 n$. Assume that the matrix of second partial derivatives $D^{2} H_{0}$ is non-degenerate in the $n$ dimension normal to $T_{0}^{n}$ in $W^{c}\left(T_{0}^{n}, H_{0}\right)$. (For system (1.7) this assumption is just that $G_{j}^{\prime \prime}\left(I_{j_{0}}\right) \neq 0$ for all $j$. Also, see conditions in $[14, \S 6]$.)

Because $W^{\mathrm{c}}\left(T_{0}^{n}, H_{0}\right)$ is normally hyperbolic by (H3), for $\varepsilon>0$ small enough there persists a (locally) invariant submanifold $W_{\varepsilon}^{\mathrm{c}}$. Also, the canonical 2 -form is necessarily non-degenerate on $W^{\mathrm{c}}\left(T_{0}^{n}, H_{0}\right)$ and so on $W_{\varepsilon}^{\mathrm{c}}$. Because of the nondegeneracy assumption ( $\mathrm{H} 4)$, the torus $T_{0}^{n}$ persists for $\varepsilon>0$ by KAM theory to give a torus $T_{\varepsilon}^{n} \subset W_{\varepsilon}^{\mathrm{c}}$ on which the motion is quasi-periodic. In fact, $T_{\varepsilon}^{n}$ depends differentiably on $\varepsilon$. In the case where $H$ is analytic, Moser proved this in $[14, \S 6]$; in the differentiable case it can be proved, for example, using the techniques in [20].

Now, for $z \in \Gamma$, let $N_{z}$ be $(n+1)$-subspace transverse to $\Gamma$ at $z$. Then, for $\varepsilon>0$, there are points $z^{\mathrm{s}}(\varepsilon)$ and $z^{\mathrm{u}}(\varepsilon)$ corresponding to $z$ given by $z^{\sigma}(\varepsilon)=N_{z} \cap$ $W^{\sigma}\left(T_{\varepsilon}^{n}, H^{\varepsilon}\right)$ for $\sigma=\mathrm{u}, \mathrm{s}$. These points vary smoothly in $\varepsilon$, because the stable and unstable manifolds vary smoothly with $\varepsilon$ on compact sets (and $T_{\varepsilon}^{n}$ varies smoothly with $\varepsilon)$. The Melnikov vector $M(z)=\left(M_{1}(z), \ldots, M_{n}(z)\right)$ is defined by

$$
\left.M_{k}(z)=\frac{\partial}{\partial \varepsilon}\left\{F_{k}\left(z^{\mathrm{u}}(\varepsilon)\right)-F_{k}\left(z^{\mathrm{s}}\right)(\varepsilon)\right)\right\}\left.\right|_{\varepsilon=0} .
$$

The vector $M(z)$ clearly measures the infinitesimal separation of $W^{\mathrm{u}}\left(T_{\varepsilon}^{n}, H^{\varepsilon}\right)$ and $W^{\mathrm{s}}\left(T_{\varepsilon}^{n}, H^{\varepsilon}\right)$ in the $n$-directions transverse to $\Gamma$ in $\left(H^{\varepsilon}\right)^{-1}(h)$. The following theorem gives an integral to calculate $M(z)$.

THEOREM 4. Let $H^{\varepsilon}(z)$ be as in (1.8) and satisfy assumptions (H1)-(H4) above. Let 
$M(z)$ be defined by (1.9). Then the components of $M$ can be calculated by the following integrals:

$$
M_{k}(z)=\lim _{j \rightarrow 0} \int_{-T_{j}^{*}}^{T_{j}}\left(D F_{k} \cdot X_{H_{1}}\right)_{\phi_{0}(t, z)} d t
$$

where $T_{j}$ and $T_{j}^{*} \rightarrow \infty$ are chosen so that the distance

$$
\lim _{j \rightarrow 0} d\left(\phi_{0}\left(-T_{j}^{*}, z\right), \phi_{0}\left(T_{j}, z\right)\right)=0 .
$$

As in theorem 1, the convergence is absolute if $D F_{j} \cdot X_{H_{1}}=0$ for points of $T_{0}^{n}$. Furthermore, if $M(z)$ has non-degenerate zero, then the system has a transverse homoclinic intersection for $\varepsilon>0$ small enough.

The easiest way to satisfy the assumptions on the $T_{j}$ and $T_{j}^{*}$ is to take the $T_{j}$ and $T_{j}^{*}$ so that $\phi_{0}\left(T_{j}, z\right)$ and $\phi_{0}\left(-T_{j}^{*}, z\right)$ both converge to the same point on $T_{0}^{n}$. For example, for system (1.7) the only condition that is necessary is that $\lim \mid \theta\left(-T_{j}^{*}, z_{0}\right)-$ $\cdot \theta\left(T_{j}, z_{0}\right) \mid=0$ in $\left(S^{1}\right)^{n}$. Since $\theta_{k}(t)=\theta_{k_{0}}+\Omega_{k} t$, where $\Omega_{k}=G_{k}^{\prime}\left(I_{j}\right)$, this means $\lim \left|\Omega_{k}\left(T_{j}-T_{j}^{*}\right)\right|=0 \bmod 2 \pi$ for all $k$. Taking $T_{j}$ and $T_{j}^{*}$ so that $\Omega_{n}\left(T_{j}-T_{j}^{*}\right)=2 \pi N_{j}$ for some integers $N_{j}$, this means we need $\lim \left|2 \pi \Omega_{k} N_{j} / \Omega_{n}\right|=0 \bmod 2 \pi$ for $1 \leq k \leq$ $n-1$. This is clearly possible by the quasi-periodicity on $T_{0}^{n}$. Also note that in Arnold's orginal paper [1] the torus did not vary with $\varepsilon$, so $D F_{k} \cdot X_{H_{1}}=0$ on $T_{0}^{n}$ and the convergence was absolute.

In [9] Holmes and Marsden mention that the integrals are not necessarily absolutely convergent, but they do not state explicitly how the times $T_{j}^{*}$ and $T_{j}$ have to be chosen so the limit is equal to $M$, i.e. so the conditionally convergent integral has the desired dynamical meaning. (It is not enough to make any choice such that the limit exists, as example 1.5 shows.) Salem also noticed this and the situation was partially clarified in [19]. The proof of this theorem is similar to theorem 1 and is discussed in $\$ 2$.

1E. Gruendler [6] has a different way of constructing a vector-valued Melnikov integral in higher dimensions and does not assume the unperturbed problem is completely integrable. We sketch his ideas. He considers a case like theorem 2 with a fixed point and periodic time perturbation

$$
\dot{x}=f(x)+h(x, t, \varepsilon),
$$

where $\varepsilon=\left(\varepsilon_{1}, \ldots, \varepsilon_{N}\right)$ and $h(x, t, 0) \equiv 0$. He considers the most general case, but we explain only the case when $f$ is Hamiltonian (or at least $\operatorname{div} f \equiv 0$ ) and $W^{\mathrm{u}}(P, f)=$ $W^{\mathrm{s}}(P, f)=\Gamma$. If $x_{0}$ is in $R^{2 n}$ (he uses $n$ ), he constructs $\left\{\psi^{(1)}\left(t, x_{0}\right), \ldots, \psi^{(2 n)}\left(t, x_{0}\right): x_{0}\right.$ is in $\Gamma$, where (i) each $\psi^{(j)}$ satisfies the variation equation $\dot{u}=D f\left(\phi_{0}\left(t, x_{0}\right)\right) u$ and (ii) $\psi^{(n+1)}, \ldots, \psi^{(2 n)}$ are tangent to $\Gamma$ and the $\psi^{(j)}\left(t, x_{0}\right)$ grow at different rates as $t$ goes to $\pm \infty$ due to the different eigenvalues at the fixed point $P$. For $x_{0}$ in $\Gamma$ let $\Pi\left(x_{0}\right)$ be the plane transverse to $\Gamma$ spanned by $\left\{\psi^{(1)}\left(0, x_{0}\right), \ldots, \psi^{(n)}\left(0, x_{0}\right)\right\}$. Let $x^{\mathrm{u}}\left(t, x_{0}, \xi, \varepsilon\right)$ be the solution of (1.1) with initial condition at $t=\xi$ given by $x^{\mathrm{u}}\left(\xi, x_{0}, \xi, \varepsilon\right) \in \Pi\left(x_{0}\right) \cap W^{\mathrm{u}}(P, f+h(\cdot, \varepsilon))$. Then functions $\Delta_{i}^{\mathrm{u}}$ are defined by

$$
x^{\mathrm{u}}\left(\xi, x_{0}, \xi, \varepsilon\right)-x_{0}=\sum_{j=1}^{n} \Delta_{i}^{\mathrm{u}}\left(x_{0}, \xi, \varepsilon\right) \psi^{(j)}\left(\xi, x_{0}\right) \text {. }
$$


If $\Omega$ is a volume, he notes that

$$
\Delta_{i}^{\mathrm{u}}\left(x_{0}, \xi, \varepsilon\right)=\Omega\left(\psi^{(1)}, \ldots, \psi^{(i-1)}, x^{\mathrm{u}}\left(\xi, x_{0}, \xi, \varepsilon\right)-x_{0}, \psi^{(i+1)}, \ldots, \psi^{(2 n)}\right),
$$

where the $\psi^{(j)}$ are evaluated at $\left(\xi, x_{0}\right)$. It is then shown that the derivative of $\Delta_{i}^{u}$ with respect to the vector $\varepsilon$ at $\varepsilon=0$ in the direction $\varepsilon$ satisfies

$$
M_{i}^{\mathrm{u}}\left(x_{0}, \xi\right) \varepsilon=D_{\varepsilon} \Delta_{i}^{\mathrm{u}}\left(x_{0}, \xi, 0\right) \varepsilon=\Omega\left(\psi^{(1)}, \ldots, \psi^{(i-1)},\left(D_{\varepsilon} x^{u}\right) \cdot \varepsilon, \psi^{(i+1)}, \ldots, \psi^{(2 n)}\right),
$$

where the $\psi^{(j)}$ are evaluated at $\left(\xi, x_{0}\right)$, and

$$
M_{i}^{\mathrm{u}}\left(x_{0}, \xi\right) \varepsilon=\int_{-\infty}^{\xi} \Omega\left(\psi^{(1)}, \ldots, \psi^{(i-1)},\left(D_{\varepsilon} h\right) \varepsilon, \psi^{(i+1)}, \ldots, \psi^{(2 n)}\right) d t
$$

where the $\psi^{(j)}$ are evaluated as

$$
\psi^{(j)}\left(t, \phi_{0}\left(t-\xi, x_{0}\right)\right) \quad \text { and } \quad\left(D_{\varepsilon} h\right) \varepsilon=\left(D_{\varepsilon} h\left(\phi_{0}\left(t-\xi, x_{0}\right), t, 0\right)\right) \varepsilon .
$$

Similarly $\Delta_{i}^{\mathrm{s}}$ is defined and $M_{i}^{\mathrm{s}}\left(x_{0}, \xi\right) \varepsilon=D_{\varepsilon} \Delta_{i}^{\mathrm{s}}\left(x_{0}, \xi, 0\right) \varepsilon$. Thus

$$
M_{i}\left(x_{0}, \xi\right) \varepsilon=\int_{-\infty}^{\infty} \Omega\left(\psi^{(1)}, \ldots, \psi^{(i-1)},\left(D_{\varepsilon} h\right) \varepsilon, \psi^{(i+1)}, \ldots, \psi^{(2 n)}\right) d t .
$$

Considering $M_{i}\left(x_{0}, \xi\right)$ as a row vector, then

$$
M\left(x_{0}, \xi\right)=\left(\begin{array}{c}
M_{1} \\
\vdots \\
M_{n}
\end{array}\right)
$$

is an $n$ by $N$ matrix. If it has rank $n$, then $n$ of the parameters $\varepsilon_{j}$ can be solved in terms of the others and still give a zero of $\Delta_{i}\left(x_{0}, \xi, \varepsilon\right)=\Delta_{i}^{\mathrm{u}}\left(x_{0}, \xi, \varepsilon\right)-\Delta_{i}^{\mathrm{s}}\left(x_{0}, \xi, \varepsilon\right)=0$ and so a homoclinic orbit [6, theorem 3.2]. If this point is a non-degenerate zero as $\left(x_{0}, \xi\right)$ varies in $\Gamma$, then the homoclinic intersection is transverse [6, theorem 4.1]. 1F. The final results of this paper turn away from different contexts in which Melnikov integrals can be defined and consider conditions which imply hyperbolic horseshoes without transverse intersections. In particular, the assumption that $M$ has a non-degenerate zero is replaced with the assumption that (a scalar-valued Melnikov integral) $M$ changes sign. The following theorem considers the case of a Hamiltonian system in four dimensions, where $X_{0}$ has a hyperbolic closed orbit with a homoclinic connection.

THEOREM 5. A. Make the assumptions of theorem 1. If $M(y)$ changes sign at $y_{0}$ as $y$ varies on $\Gamma=\left[W^{\mathrm{s}}\left(\gamma, H_{0}\right)-\gamma\right] \cap W^{\mathrm{u}}\left(\gamma, H_{0}\right)$, then, for $\varepsilon>0$ small enough, the manifolds $W^{\mathrm{s}}\left(\gamma_{\varepsilon}, H^{\varepsilon}\right)$ and $W^{\mathrm{u}}\left(\gamma_{\varepsilon}, H^{\varepsilon}\right)$ have a topologically transverse intersection near $y_{0}$ (they cross each other).

B. If $H^{\varepsilon}(z)$ is analytic in $z$, then, for $\varepsilon>0$ small enough, the system has hyperbolic horseshoes arbitrarily near these topologically transverse intersections and is nonintegrable.

The only reason $H^{\varepsilon}(z)$ needs to be analytic in part $\mathrm{B}$ is to be able to conclude that the original topologically transverse intersection, corresponding to a zero of $M$, has finite order of contact. 
Several people have noted that theorem $5 \mathrm{~B}$ is true, including de Carvalho and Roussarie [2], Conley as given in [3], and probably others. Most of these papers prove the result by showing that arbitrarily near the intersection of finite order these are necessarily (differentiably) transverse intersections. It also follows by a direct proof following [18, proposition 5.1]. A sketch of this later proof is given in $\S 4$ for the case of a hyperbolic fixed point considered in theorem 6 below. This proof is essentially (implicitly) contained in [18] for the case of theorem 5B. That paper, in turn, is a direct evolution of the theorems and methods of Newhouse [15]. Earlier, Gavrilov and Silnikov [5] had used some of these ideas in their work. Some related ideas appear in the work on bifurcations from Morse/Smale systems by Newhouse and Palis [16]. They consider what bounds on the angles of intersections of stable and unstable manifolds are sufficient to give a hyperbolic horseshoe. Also, Patterson considered some questions related to theorem 5 in his thesis [17]. I had several useful conversations with him about ideas related to these before this paper was written.

Similar ideas for non-transverse intersections can be applied to the case of hyperbolic fixed point with non-zero imaginary part of the eigenvalues. When the manifolds are transverse, Devaney proved there is a hyperbolic horseshoe [4]. In fact, if the system has a topologically transverse homoclinic intersection with finite-order contact, then the following theorem states the same result holds.

THEOREM 6. A. Let $H$ be an analytic Hamiltonian function in dimension four with a hyperbolic fixed $P$ point whose eigenvalues have non-zero imaginary parts. If $W^{\mathrm{u}}(P, H)$ and $W^{\mathrm{s}}(P, H)$ have a topologically transverse intersection at $z_{0}$ in $H^{-1}\left(h_{0}\right)$, with $h_{0}=H(P)$, then there is a horseshoe arbitrarily near $z_{0}$.

B. Let $H^{\varepsilon}=H_{0}+\varepsilon H_{1}$ be as in theorem 2. If $M(z)$ changes sign at $z_{0}=\Gamma=$ $W^{\mathrm{s}}\left(P, H_{0}\right) \cap W^{\mathrm{u}}\left(P, H_{0}\right)$, then, for $0<\varepsilon \leq \varepsilon_{0}$ small enough, the vector field $X^{\varepsilon}$ for $H^{\varepsilon}$ has a horseshoe arbitrarily near $z_{0}$.

The proof of theorem $6 \mathrm{~A}$ combines the methods of [4] and [18] and is sketched in $\S 4$. Theorem $6 \mathrm{~B}$ follows because $M$ gives the lowest-order terms in $\varepsilon$.

\section{Proof of theorem 1}

In the definition of $M(z)$, we need to calculate

$$
\left.\frac{\partial}{\partial \varepsilon}\left(F \circ z^{\sigma}(\varepsilon)\right)\right|_{\varepsilon=0} \quad \text { for } \sigma=\text { u, s. }
$$

Instead of merely calculating this, we calculate

$$
\left.\frac{\partial}{\partial \varepsilon}\left(F \circ \phi\left(t, z^{\sigma}(\varepsilon), \varepsilon\right)\right)\right|_{\epsilon=0}
$$

and indeed

$$
\left.\frac{d}{d t} \frac{\partial}{\partial \varepsilon}\left(F \circ \phi\left(t, z^{\sigma}(\varepsilon), \varepsilon\right)\right)\right|_{\varepsilon=0^{\circ}}
$$


Proceeding for $\sigma=\mathrm{u}, \mathrm{s}$, taking all derivatives at $\varepsilon=0$,

$$
\begin{aligned}
\frac{d}{d t} \frac{\partial}{\partial \varepsilon} F \circ \phi\left(t, z^{\sigma}(\varepsilon), \varepsilon\right) & =\frac{\partial}{\partial \varepsilon} \frac{d}{d t} F \circ \phi\left(t, z^{\sigma}(\varepsilon), \varepsilon\right) \\
& =\frac{\partial}{\partial \varepsilon}\left(D F \cdot X^{\varepsilon}\right)_{\phi\left(t, z^{\sigma}(\varepsilon), \varepsilon\right)} \\
& =\left(D F \cdot X_{1}\right)_{\phi_{0}(t, z)}+\frac{\partial}{\partial \varepsilon}\left(D F \cdot X_{0}\right)_{\phi\left(t, z^{\sigma}(\varepsilon), \varepsilon\right)} \\
& =\left(D F \cdot X_{1}\right)_{\phi_{0}(t, z)} .
\end{aligned}
$$

The first term on the third line comes from differentiating the $\varepsilon$ in $X^{\varepsilon}$ and putting $\varepsilon=0$ elsewhere, while the second term is the reverse. The second term is zero because $D F \cdot X_{0} \equiv 0$ at all points. Integrating this from $T_{1}$ to $T_{2}$ gives

$$
\frac{\partial}{\partial \varepsilon} F \circ \phi\left(T_{2}, z^{\sigma}(\varepsilon), \varepsilon\right)-\frac{\partial}{\partial \varepsilon} F \circ \phi\left(T_{1}, z^{\sigma}(\varepsilon), \varepsilon\right)=\int_{T_{1}}^{T_{2}}\left(D F \cdot X_{1}\right)_{\phi_{0}(t, z)} d t .
$$

For $\sigma=\mathrm{u}$ take $T_{1}=-T_{j}^{*}$ and $T_{2}=0$, while for $\sigma=\mathrm{s}$ take $T_{1}=0$ and $T_{2}=T_{j}$. Adding the two terms gives

$$
\begin{aligned}
M(z)= & \int_{-T_{j}^{*}}^{0}\left(D F \cdot X_{1}\right)_{\phi_{0}(t, z)} d t+\int_{0}^{T_{j}}\left(D F \cdot X_{1}\right)_{\phi_{0}(t, z)} d t \\
& +\frac{\partial}{\partial \varepsilon} F \circ \phi\left(-T_{j}^{*}, z^{\mathrm{u}}(\varepsilon), \varepsilon\right)-\frac{\partial}{\partial \varepsilon} F \circ \phi\left(T_{j}, z^{\mathrm{s}}(\varepsilon), \varepsilon\right) .
\end{aligned}
$$

Taking the limit as $j \rightarrow \infty$, it is only necessary to prove the limit of the sum of the last two terms goes to zero with the choice of time $-T_{j}^{*}$ and $T_{j}$. The intuitive reason is that $\phi\left(-T_{j}^{*}, z_{0}, 0\right)$ and $\phi\left(T_{j}, z_{0}, 0\right)$, for $z_{0}=z^{\mathrm{u}}(0)=z^{\mathrm{s}}(0)$, go to the same point on the closed orbit $\gamma$, and this term measures the displacement of $\gamma$ in terms of the change of values of $F$. Proceeding more carefully, the following lemma completes the proof of theorem 1 .

LEMMA 2.1

$$
\lim _{j \rightarrow \infty}\left(\frac{\partial}{\partial \varepsilon} F \circ \phi\left(-T_{j}^{*}, z^{\mathrm{u}}(\varepsilon), \varepsilon\right)-\frac{\partial}{\partial \varepsilon} F \circ \phi\left(T_{j}, z^{\mathrm{s}}(\varepsilon), \varepsilon\right)\right)=0
$$

with $T_{j}$ and $T_{j}^{*}$ chosen as in theorem 1.

Before proving lemma 2.1, we prove the special case of theorem 4 assuming $H^{\varepsilon}$ is given by (1.7), where the explicit variables make the exposition simpler. The first part of the proof of theorem 4 is exactly like the proof above, where $K$ is now either the integral $F$ or $I_{1}, \ldots, I_{n-1}$. Given $y_{0}$ a homoclinic point for $F$ and any $\theta_{0}=\left(\theta_{10}, \ldots, \theta_{n 0}\right)$, there is a point on the stable or unstable manifold $z^{\mathrm{s}}\left(\theta_{0}, \varepsilon\right)$ and $z^{\mathrm{u}}\left(\theta_{0}, \varepsilon\right)$ for $\varepsilon>0$. Also, the invariant torus for $\varepsilon>0$ can be written as a function (or graph) $z=\psi(\theta, \varepsilon)=\psi^{E}(\theta)$. The lemma which corresponds to 2.1 is the following:

LEMMA 2.2

$$
\lim _{j \rightarrow \infty}\left|\frac{\partial}{\partial \varepsilon} K \circ \phi\left(-T_{j}^{*}, z^{\mathrm{u}}\left(\theta_{0}, \varepsilon\right), \varepsilon\right)-\frac{\partial}{\partial \varepsilon} K \circ \phi\left(T_{j}, z^{\mathrm{s}}\left(\theta_{0}, \varepsilon\right), \varepsilon\right)\right|=0
$$


if $\lim _{j \rightarrow \infty} \mid \theta\left(T_{j}^{*}, z\left(\theta_{0}, 0\right)\right)-\theta\left(T_{j}, z\left(\theta_{0}, 0\right) \mid=0\right.$. Here $\theta\left(t, z_{0}\right)$ is the $\theta$-component of the flow for Hamiltonian (1.7) with $\varepsilon=0$ and initial conditions $z_{0}$.

Proof. We want to determine the nature of the limit of the vector $(\partial / \partial \varepsilon) \phi\left(T_{j}, z^{s}\left(\theta_{0}, \varepsilon\right), \varepsilon\right)$. In fact we want to decompose it into the component along the invariant torus and the normal component. To clarify the convergence, it is necessary to extend the flow into $(z, \varepsilon)$-space with $\dot{\varepsilon}=0$. Label this flow as $\phi^{*}\left(t, z_{0}, \varepsilon\right)$. Also let $\theta\left(t, z_{0}, \varepsilon\right)$ be the $\theta$-coordinate of the flow $\phi^{*}\left(t, z_{0}, \varepsilon\right)$ and $\theta\left(t, z_{0}\right)=\theta\left(t, z_{0}, 0\right)$. Let $\gamma=\{\psi(\theta, 0)\}$ be the original unperturbed torus and $\gamma^{*}=$ $\{(\psi(\theta, \varepsilon), \varepsilon)\}$ be the extended perturbed torus with stable manifold $W^{s}\left(\gamma^{*}, H^{\varepsilon}\right) \subset$ $\{(z, \varepsilon)\}$.

The vector $(\partial / \partial \varepsilon)\left(z^{s}\left(\theta_{0}, \varepsilon\right), \varepsilon\right)$ is tangent to $W^{s}\left(\gamma^{*}, H^{\varepsilon}\right)$ and so it follows that

$$
\frac{\partial}{\partial \varepsilon} \phi^{*}\left(T_{j}, z^{\mathrm{s}}\left(\theta_{0}, \varepsilon\right), \varepsilon\right)=\left(D_{z} \phi_{\left(T_{j}, z_{0}, 0\right)} \frac{\partial}{\partial \varepsilon} z^{\mathrm{s}}\left(\theta_{0}, \varepsilon\right), 1\right)
$$

is also. Because $\gamma^{*}$ is normally attracting in $W^{\mathrm{s}}\left(\gamma^{*}, H^{\varepsilon}\right)$, as $T_{j}$ goes to infinity the component of the vector normal to $\gamma^{*}$ goes to zero. Proceeding with explicit calculations,

$$
\begin{aligned}
\lim _{j \rightarrow \infty}( & \left.\frac{\partial}{\partial \varepsilon} K \circ \phi\left(-T_{j}^{*}, z^{\mathrm{u}}\left(\theta_{0}, \varepsilon\right), \varepsilon\right)-\frac{\partial}{\partial \varepsilon} K \circ \phi\left(T_{j}, z^{\mathrm{s}}\left(\theta_{0}, \varepsilon\right), \varepsilon\right)\right) \\
= & \lim \left(D K \frac{\partial}{\partial \varepsilon}\left[\phi\left(-T_{j}^{*}, z^{\mathrm{u}}\left(\theta_{0}, \varepsilon\right), \varepsilon\right)\right]-D K \frac{\partial}{\partial \varepsilon}\left[\psi\left(\theta\left(-T_{j}^{*}, z^{\mathrm{u}}\left(\theta_{0}, \varepsilon\right), \varepsilon\right), \varepsilon\right)\right]\right) \\
& +\lim \left(D K \frac{\partial}{\partial \varepsilon}\left[\psi\left(\theta\left(-T_{j}^{*}, z^{\mathrm{u}}\left(\theta_{0}, \varepsilon\right), \varepsilon\right), \varepsilon\right)\right]-D K \frac{\partial}{\partial \varepsilon}\left[\psi\left(\theta\left(-T_{j}^{*}, z_{0}, 0\right), \varepsilon\right)\right]\right) \\
& +\lim \left(-D K \frac{\partial}{\partial \varepsilon}\left[\phi\left(T_{j}, z^{\mathrm{s}}\left(\theta_{0}, \varepsilon\right), \varepsilon\right)\right]+D K \frac{\partial}{\partial \varepsilon}\left[\psi\left(\theta\left(T_{j}, z^{\mathrm{s}}\left(\theta_{0}, \varepsilon\right), \varepsilon\right), \varepsilon\right)\right]\right) \\
& +\lim \left(-D K \frac{\partial}{\partial \varepsilon}\left[\psi\left(\theta\left(T_{j}, z^{\mathrm{s}}\left(\theta_{0}, \varepsilon\right), \varepsilon\right), \varepsilon\right)\right]+D K \frac{\partial}{\partial \varepsilon}\left[\psi\left(\theta\left(T_{j}, z_{0}, 0\right), \varepsilon\right)\right]\right) \\
& +\lim \left(D K \frac{\partial}{\partial \varepsilon}\left[\psi\left(\theta\left(-T_{j}^{*}, z_{0}, 0\right), \varepsilon\right)\right]-D K \frac{\partial}{\partial \varepsilon}\left[\psi\left(\theta\left(T_{j}, z_{0}, 0\right), \varepsilon\right)\right]\right) .
\end{aligned}
$$

The fifth limit is zero because of the choice of $T_{j}^{*}$ and $T_{j}$.

We consider only the third and fourth limits because the first two are similar. Considering the third limit, the vector

$$
\frac{\partial}{\partial \varepsilon} \phi^{*}\left(T_{j}, z^{\mathrm{s}}\left(\theta_{0}, \varepsilon\right), \varepsilon\right)=D \phi_{\left(T_{j}, z_{0}, 0\right)}^{*}\left(\frac{\partial}{\partial \varepsilon} z^{\mathrm{s}}\left(\theta_{0}, \varepsilon\right), 1\right)
$$

is tangent to $W^{\mathrm{s}}\left(\gamma^{*}, \dot{H}^{\varepsilon}\right)$. As $T_{j}$ goes to infinity, the component of this vector normal to $\gamma^{*}$ goes to zero (by the contraction on this manifold), and further the two vectors

$$
\frac{\partial}{\partial \varepsilon} \phi^{*}\left(T_{j}, z^{\mathrm{s}}\left(\theta_{0}, \varepsilon\right), \varepsilon\right) \quad \text { and } \quad \frac{\partial}{\partial \varepsilon} \psi\left(\theta\left(T_{j}, z^{\mathrm{s}}\left(\theta_{0}, \varepsilon\right), \varepsilon\right), \varepsilon\right)
$$

have the same components along $\gamma^{*}$, so the third limit is zero. 
Finally, for the fourth limit,

$$
\frac{\partial}{\partial \varepsilon} \psi\left(\theta\left(T_{j}, z^{\mathrm{s}}\left(\theta_{0}, \varepsilon\right), \varepsilon\right), \varepsilon\right)-\frac{\partial}{\partial \varepsilon} \psi\left(\theta\left(T_{j}, z_{0}, 0\right), \varepsilon\right)=\frac{\partial \psi^{0}}{\partial \theta} \frac{\partial}{\partial \varepsilon} \theta\left(T_{j}, z^{\mathrm{s}}\left(\theta_{0}, \varepsilon\right), \varepsilon\right)
$$

This last vector is tangent to $\gamma$ (not just $\gamma^{*}$ ), and so acting on it by $D K$ gives zero. Thus the fourth limit is zero. This completes the proof of lemma 2.2.

Proof of lemma 2.1. The main difference in this proof is that there is no explicit coordinate corresponding to $\theta$ in the proof of lemma 2.2 which can be used to associate a point on the closed orbit $\gamma$ or $\gamma^{*}$. However, the stable manifold theorem says that points on $W^{s}\left(\gamma^{*}, H^{\varepsilon}\right)$ approach $\gamma^{*}$ in phase with some point on $\gamma^{*}$, i.e. if $\left(z_{\varepsilon}, \varepsilon\right)$ is on $W^{s}\left(\gamma^{*}, H^{\varepsilon}\right)$, then there is a point $\left(z_{1}, \varepsilon\right)$ on $\gamma^{*}$ such that $\mid \phi^{*}\left(t, z_{\varepsilon}, \varepsilon\right)-$ $\phi^{*}\left(t, z_{1}, \varepsilon\right) \mid$ goes to zero as $t$ goes to infinity. We use this to define a continuous function

$$
\pi_{1}: W^{s}\left(\gamma^{*}, H^{\varepsilon}\right) \rightarrow \gamma^{*}
$$

by

$$
\pi_{1}\left(z_{\varepsilon}, \varepsilon\right)=\left(z_{1}, \varepsilon\right)
$$

and

$$
\pi: W^{\mathrm{s}}\left(\gamma^{*}, H^{\varepsilon}\right) \rightarrow \gamma
$$

by

$$
\pi\left(z_{\varepsilon}, \varepsilon\right)=\psi^{-1} \pi_{1}\left(z_{\varepsilon}, \varepsilon\right),
$$

where $\{\psi(z, \varepsilon): z$ in $\gamma)\}=\gamma_{\varepsilon} \times\{\varepsilon\}$. Then in equation (2.3) $\theta(t, z, \varepsilon)$ is replaced by $\pi \circ \phi^{*}(t, z, \varepsilon)$ and the initial condition is specified by $z_{0}$ and not just $\theta_{0}$, e.g. $\psi\left(\theta\left(T_{j}, z^{\mathrm{s}}\left(\theta_{0}, \varepsilon\right), \varepsilon\right), \varepsilon\right)$ is replaced by $\psi\left(\pi^{\circ} \phi^{*}\left(T_{j}, z^{\mathrm{s}}\left(z_{0}, \varepsilon\right), \varepsilon\right), \varepsilon\right)$. The rest of the proof is the same.

General case of theorem 4. Again the first part of the proof is exactly as before but $F_{k}$ replaces $F$. The calculation of the limit is like the proof of lemma 2.1 except that the closed orbit $\gamma$ is replaced by the torus $T_{0}^{n}$ and $\pi$ is a projection onto $T_{0}^{n}$. The rest of the proof is the same.

\section{Equivalence of two integrals: theorem 3}

We start with the formula for $M(z)$. In this case the coordinates are $z=(y, \theta, I)$ and the second integral is $F(z)=I$. If $X_{1}$ is the vector field for $H_{1}$, then $D F \cdot X_{1}=$ $-\partial H_{1} / \partial \theta$. We want to compare this with $(d / d t) H_{1} \cdot \phi_{0}\left(t, z_{0}\right)$ :

$$
\frac{d}{d t} H_{1} \circ \phi_{0}\left(t, z_{0}\right)=D_{y} H_{1} \cdot X_{0}+\frac{\partial H_{1}}{\partial \theta} \cdot \dot{\theta}+\frac{\partial H_{1}}{\partial I} \cdot \dot{I},
$$

where $D_{y}$ is the partial derivative with respect to $y$ variables. But $\dot{\theta}=\partial H_{0} / \partial I=\Omega$ and $\dot{I}=0$. Also $D_{y} H_{1} \cdot X_{0}=-D_{y} H_{0} \cdot X_{1}$ either by writing out or using the result for Poisson brackets. Combining and solving for $-\partial H_{1} / \partial \theta$ in (3.1) gives

$$
D F \cdot X_{1}=-\frac{\partial H_{1}}{\partial \theta}=-\frac{1}{\Omega} D_{y} H_{0} \cdot X_{1}-\frac{1}{\Omega} \frac{d}{d t} H_{1} \circ \phi_{0}\left(t, z_{0}\right)
$$


Thus

$$
M\left(z_{0}\right)=\lim _{j \rightarrow \infty}-\int_{-T_{j}}^{T_{j}} \frac{1}{\Omega} D_{y} H_{0} \cdot X_{1} d t+\int_{-T_{j}^{*}}^{T_{j}}-\frac{1}{\Omega} \frac{d}{d t}\left(H_{1} \circ \phi_{0}\left(t, z_{0}\right)\right) d t .
$$

Using integration by parts in the second integral with $u=-1 / \Omega$ and $d v=$ $(d / d t)\left(H_{1} \circ \phi_{0}(t, z)\right) d t$ gives

$$
d u=\frac{1}{\Omega^{2}}\left(D_{y} \Omega \cdot X_{0}+\frac{\partial \Omega}{\partial I} \dot{I}+\frac{\partial \Omega}{\partial \theta} \dot{\theta}\right) d t=\frac{1}{\Omega^{2}} D_{y} \Omega \cdot X_{0} d t=-\frac{1}{\Omega^{2}} D_{y} H_{0} \cdot X_{\Omega} d t
$$

because $\dot{I}=0, \partial \Omega / \partial \theta=0$ and $D_{y} \Omega \cdot X_{0}=-D_{y} H_{0} \cdot X_{\Omega}$. Thus

$$
\begin{aligned}
M\left(z_{0}\right) & =\lim _{j \rightarrow \infty}-\int_{-T_{j}^{*}}^{T_{j}} D_{y} H_{0}\left(\frac{X_{1}}{\Omega}-\frac{H_{1}}{\Omega^{2}} X_{\Omega}\right) d t-\left.\lim _{j \rightarrow \infty} \frac{1}{\Omega} H_{1} \circ \phi_{0}\left(t, z_{0}\right)\right|_{-T_{j}^{*}} ^{T_{j}} \\
& =\int_{-\infty}^{\infty} D_{y} H_{0} \cdot Y_{1} d t .
\end{aligned}
$$

The boundary terms go to zero by the choice of $T_{j}$ and $T_{j}^{*}$.

\section{Proof of theorem 6}

The proof combines the ideas of the proof of Devaney [4] with the ideas when the intersection is only topologically transverse at a homoclinic intersection for a diffeomorphism in two dimensions [18, propositions 5.1 and 7.8].

Following Devaney, there are coordinates $(x, y)=\left(x_{1}, x_{2}, y_{1}, y_{2}\right)$ near the fixed point $P=(0,0)$ where the flow is given by

$$
F_{t}(x, y)=\left(\begin{array}{c}
e^{A t}\left(x_{1} \cos \Omega t-x_{2} \sin \Omega t\right) \\
e^{A t}\left(x_{1} \sin \Omega t+x_{2} \cos \Omega t\right) \\
e^{-A t}\left(y_{1} \cos \Omega t+y_{2} \sin \Omega t\right) \\
e^{-A t}\left(-y_{1} \sin \Omega t+y_{2} \cos \Omega t\right)
\end{array}\right)
$$

where $A=A(x, y)$ and $\Omega=\Omega(x, y)$ are analytic functions of the initial conditions $(x, y)$. For small enough $r, \delta$, there are transversals to the flow near the local unstable and stable manifolds:

$$
\begin{aligned}
& \Sigma^{\mathbf{u}}=\{(x, y):|x|=r,|y| \leq \delta\}, \\
& \Sigma^{\mathbf{s}}=\{(x, y):|x| \leqslant \delta,|y|=r\} .
\end{aligned}
$$

Assuming $H(P)=0$, let $\Sigma_{0}^{\mathrm{s}}=\Sigma^{\mathrm{s}} \cap H^{-1}(0)$ and $\Sigma_{0}^{\mathrm{u}}=\Sigma^{\mathrm{u}} \cap H^{-1}(0)$. Also let $\sigma^{\mathrm{s}}=\Sigma_{0}^{\mathrm{s}} \cap$ $\{x=0\}$ (resp. $\sigma^{u}=\Sigma_{0}^{u} \cap\{y=0\}$ ) be the intersection of the local stable (resp. unstable) manifold with $\Sigma_{0}^{\mathrm{s}}$ (resp. $\Sigma_{0}^{u}$ ). If $\gamma$ is the orbit of the homoclinic intersection given in the theorem, let $q^{s}=\gamma \cap \sigma^{s}$ and $q^{u}=\gamma \cap \sigma^{u}$. Let $D^{s}$ and $D^{u}$ be two-dimensional discs in $\Sigma_{0}^{s}$ and $\Sigma_{0}^{u}$ centred at $q^{\mathrm{s}}$ and $q^{\mathrm{u}}$.

There are Poincaré maps defined from points near $q^{\mathrm{s}}$ to itself (at least for some points). This map is the composition of two maps, $\Phi_{0}:\left(D^{\mathrm{s}}-\sigma^{\mathrm{s}}\right) \rightarrow \Sigma_{0}^{\mathrm{u}}$ and $\Phi_{1}: D^{\mathrm{u}} \rightarrow$ $D^{s}$. Here $\Phi_{1}$ is formed by following trajectories from points in $D^{u}$ near $q^{u}$ until they return to $\Sigma_{0}^{s}$ near $q^{s}$ and so in $D^{s}$ (if $D^{\mathrm{u}}$ is small enough). Also any point on $D^{\mathrm{s}}-\sigma^{\mathrm{s}}$ will flow past $P$ until it exists at some point in $\Sigma_{0}^{\mathrm{u}}$; this defines $\Phi_{0}$. If $\mathscr{D}=\Phi_{0}^{-1}\left(D^{\mathrm{u}}\right)$, then $\Phi=\Phi_{1} \circ \Phi_{0}: \mathscr{D} \subset\left(D^{\mathrm{s}}-\sigma^{\mathrm{s}}\right) \rightarrow D^{\mathrm{s}}$. 
Let $(\theta, z)=\left(\theta^{\mathrm{s}}, z^{\mathrm{s}}\right)$ be a coordinate system on $D^{\mathrm{s}}$ where $\sigma^{\mathrm{s}}=\left\{z^{\mathrm{s}}=0\right\}$ and $\theta^{\mathrm{s}}$ is an angular coordinate along $\sigma^{\mathrm{s}}$ with $q^{\mathrm{s}}$ corresponding to $\left(z^{\mathrm{s}}, \theta^{\mathrm{s}}\right)=(0,0)$. If the intersection is topologically transverse, then $W^{\mathrm{u}}(P, H) \cap D^{\mathrm{s}}$ is given by the graph of a function $z=a \theta^{j}+h(\theta)$, where $h(\theta)=\mathrm{O}\left(|\theta|^{j+1}\right)$. The slope of $W^{\mathrm{u}}(P, H)$ at a point $(\theta, z)$ on the graph is about $j a \theta^{j-1} \approx j a(z / a)^{(j-1) / j}=j a^{1 / j} z^{1-1 / j} \equiv \xi(z)$. For $\eta>1$ near enough to 1 , we can consider the sectors of vectors whose slopes are about $\xi(z)$,

$$
S(\theta, z)=\left\{v=\left(v_{\mathrm{s}}, v_{\mathrm{u}}\right): \eta^{-1}<\left|v_{\mathrm{u}}\right| /\left(\left|v_{\mathrm{s}}\right| \xi(z)\right)<\eta\right\} .
$$

Thus for $v$ in $S(\theta, z)$ the slope of $v$ is between $\eta^{-1} \xi(z)$ and $\eta \xi(z)$. For $v$ in $S(\theta, z)$ let $v^{\prime}=\left(D \Phi_{0}\right) v$ and $v^{\prime \prime}=(D \Phi) v=\left(D \Phi_{1}\right) v^{\prime}$. We show that for $v$ in $S(\theta, z)$ the vector of $v^{\prime \prime}$ is also in $S(\Phi(\theta, z))$ and $\left\|v^{\prime \prime}\right\|>\|v\|$. Thus there is a hyperbolic structure.

If $v$ is a vector in $S(\theta, z)$ with $v_{s}=1$, then $\left|v_{\mathrm{u}}\right| \approx j a z^{1-1 / j}$. The calculation in [1, p. 435] shows that this component is stretched by a factor of $1 /|z|$ by the time it reaches $\Sigma_{0}^{u},\left|v_{u}^{\prime}\right| \sim|z|^{1-1 / j} \cdot|z|^{-1}=|z|^{-1 / j}$. This component is arbitrarily large as $|z| \rightarrow 0$. Also the stable component is contracted as it goes past $P$, so $\left|v_{\mathrm{s}}^{\prime}\right|<\left|v_{\mathrm{s}}\right|=1$. Thus $v^{\prime}$ is a vector pointing nearly parallel to the unstable direction. Then $v^{\prime \prime}=D \Phi v^{\prime}$ is arbitrarily close to the direction of $W^{\mathrm{u}}(P, H) \cap D^{\mathrm{s}}$ and so it lies in the sector $S(\Phi(\theta, z))$. Also $\left\|v^{\prime \prime}\right\| \geq C_{1}\left\|v^{\prime}\right\| \geq C_{1}\left|v_{\mathrm{u}}^{\prime}\right| \geq C_{2}|z|^{-1 / j}\|v\|$. As $|z| \rightarrow 0, C_{2}|z|^{-1 / j} \rightarrow \infty$, so the vector is stretched by an arbitrarily large factor when it returns to $D^{\mathrm{s}}$. (On the other hand the length of time needed to return is proportional to $|z|^{-1}$, so the characteristic exponent is proportional to $|z|^{1-1 / j}$ and goes to zero as $|z| \rightarrow 0$.) Dually the vectors in the stable direction are contracted by a similar factor (or expanded by this factor as the solutions are followed backward).

As discussed in [4], $D^{\mathrm{s}} \cap \Phi\left(D^{\mathrm{s}}\right)$ is made up of infinitely many vertical strips which accumulate on $W^{\mathrm{u}}(P, H) \cap D^{\mathrm{s}}$. Similarly, $D^{\mathrm{s}} \cap \Phi^{-1}\left(D^{\mathrm{s}}\right)$ is made up of infinitely many horizontal strips which accumulate on $\sigma^{\mathrm{s}}$. If only finitely many of these are used of each type, then there is a hyperbolic invariant set, a horseshoe. As the number of symbols increases, the hyperbolicity of $\Phi$ actually increases on these new orbits, but the hyperbolicity relative to the period of the flow (the characteristic exponents) goes to zero.

Remark. In connection with the situation in theorem $6 \mathrm{~A}$, if $H$ is completely integrable and $W^{\mathrm{s}}(P, H)=W^{\mathrm{u}}(P, H)$, then the above proof produces strips near the homoclinic connection which are mapped across themselves by the first return map. Thus it at first appears that $H$ has a hyperbolic horseshoe, which is impossible because it is completely integrable. However, the map of these strips across themselves in this case is a sheer map, so there is no hyperbolic horseshoe and there is no contradiction.

\section{REFERENCES}

[1] V. I. Arnold. Instability of dynamical systems with many degrees of freedom. Sov. Math. Dokl. 5 (1963), 581-585.

[2] S. de Carvalho \& $R$. Roussarie. Some remarks about homoclinic points of second order differential equations. Geometric Dynamics, ed. J. Palis. Lecture Notes in Mathematics 1007. Springer-Verlag, New York (1983), 88-95.

[3] R. Churchill \& D. Rod. Pathology in dynamical systems III: analytic Hamiltonians. J. Differential Equations 37 (1980), 23-38. 
[4] R. Devaney. Homoclinic orbits in Hamiltonian systems. J. Differential Equations 21 (1976), 431-438.

[5] N. K. Gavrilov \& L. P. Silnikov. On the three dimensional dynamical systems close to a system with a structurally unstable homoclinic curve I. Math. USSR Sbornik 17 (1972), 467-485; Il. Math. USSR Sbornik 19 (1973), 139-156.

[6] J. Gruendler. The existence of homoclinic orbits and the method of Melnikov for systems in $R^{\prime \prime}$. SIAM J. Math. Anal. 16 (1985), 907-931.

[7] J. Guckenheimer \& P. Holmes. Nonlinear Oscillations, Dynamical Systems, and Bifurcation of Vector Fields. Springer-Verlag, New York/Berlin/Heidelberg/Tokyo (1983).

[8] P. Holmes and J. Marsden. Horseshoes in perturbations of Hamiltonian systems with two degrees of freedom. Commun. Math. Phys. 82 (1982), 523-544.

[9] P. Holmes \& J. Marsden. Melnikov's method and Arnold diffusion for perturbation of integrable Hamiltonian systems. J. Math. Phys. 23 (1982), 669-675.

[10] P. Holmes \& J. Marsden. Horseshoes and Arnold diffusion for Hamiltonian systems on Lie groups. Indiana Univ. Math. J. 32 (1983), 273-309.

[11] L. Lerman \& Ia. Umanskii. On the existence of separatrix loops in four-dimensional systems similar to the integrable Hamiltonian systems. Appl. Math. Mech. 47 (1984), 335-340.

[12] J. Marsden. Chaos in dynamical systems by the Poincaré-Melnikov-Arnold method. Chaos in Nonlinear Dynamical Systems, ed. J. Chandra. SIAM, Philadelphia (1984), 19-31.

[13] V. K. Melnikov. On the stability of the center for time periodic perturbations. Trans. Moscow Math. Soc. 12 (1963) 1-57.

[14] J. Moser. Convergent series expansion for quasi-periodic motion. Math. Annalen 169 (1967), 136-176.

[15] S. Newhouse. Diffeomorphisms with infinitely many sinks. Topology 13 (1974), 9-18.

[16] S. Newhouse \& J. Palis. Cycles and bifurcation theory. Asterisque 31 (1976), 43-140.

[17] S. Patterson. $\Omega$-stable limit set explosions. Trans. Amer. Math. Soc. 294 (1986), 775-798.

[18] C. Robinson. Bifurcation to infinitely many sinks. Commun. Math. Phys. 90 (1983), 433-459.

[19] F. Salem, J. Marsden \& P. Varaiya. Arnold diffusion in the swing equations of a power system. To appear.

[20] E. Zehnder. Moser's implicit function theorem in the framework of analytic smoothing. Math. Annalen 219 (1976), 105-121. 